\title{
Correlation of spontaneous and traumatic anterior skull base CSF leak flow rates with fluid pattern on early, delayed, and subtraction volumetric extended echo train T2-weighted MRI
}

\author{
John W. Rutland, BA, ${ }^{1}$ Satish Govindaraj, MD, ${ }^{2}$ Corey M. Gill, BA, BS, ${ }^{1}$ Michael Shohet, MD, ${ }^{2}$ \\ Alfred M. C. Iloreta Jr., MD, ${ }^{2}$ Joshua B. Bederson, MD, ${ }^{1}$ Raj K. Shrivastava, MD, ${ }^{1}$ and \\ Bradley N. Delman, MD, MS ${ }^{3}$
}

Departments of ${ }^{1}$ Neurosurgery, ${ }^{2}$ Otolaryngology-Head and Neck Surgery, and ${ }^{3}$ Diagnostic, Molecular, and Interventional Radiology, Icahn School of Medicine at Mount Sinai, New York, New York

\begin{abstract}
OBJECTIVE CSF leakage is a potentially fatal condition that may result when a skull base dural defect permits CSF communication between the cranial vault and sinonasal cavities. Flow rate is an important property of CSF leaks that can contribute to surgical decision-making and predispose patients to complications and inferior outcomes. Noninvasive preoperative prediction of the leak rate is challenging with traditional diagnostic tools. The present study compares fluid configurations on early and late volumetric extended echo train T2-weighted MRI by using image tracings and sequence subtraction as a novel method of quantifying CSF flow rate, and it correlates radiological results with intraoperative findings and clinical outcomes.
\end{abstract}

METHODS A total of 45 patients met inclusion criteria for this study and underwent 3-T MRI. Imaging sequences included two identical CUBE T2 (vendor trade name for volumetric extended echo train T2) acquisitions at the beginning and end of the scanning session, approximately 45 minutes apart. Twenty-five patients were confirmed to have definitive spontaneous or traumatic anterior skull base CSF leaks. Semiautomated volumetric segmentation of CSF intensity was performed on both CUBE data sets by using 3D-Slicer software, and volumes were subtracted to obtain accumulated CSF volume. These imaging-derived fluid accumulations were correlated with high- or low-flow states, as well as ultimate treatment outcomes including recurrences.

RESULTS Of the 45 patients, 25 (55.6\%) had definitive evidence of CSF leakage, and $22(88 \%)$ of these underwent surgical repair. Patients with high-flow CSF leaks had higher early $\left(4.058 \mathrm{~cm}^{3} \mathrm{vs} 0.982 \mathrm{~cm}^{3}, p=0.04\right)$, late $\left(4.58 \mathrm{~cm}^{3} \mathrm{vs}\right.$ $\left.1.096 \mathrm{~cm}^{3}, p=0.04\right)$, and accumulated $\left(0.53 \mathrm{~cm}^{3} \mathrm{vs} 0.11 \mathrm{~cm}^{3}, p=0.01\right)$ fluid volume measurements than patients with low-flow leaks. The $5(22.7 \%)$ patients who exhibited postoperative CSF leak recurrence had significantly greater early $\left(6.30 \mathrm{~cm}^{3}\right.$ vs $\left.1.23 \mathrm{~cm}^{3}, p=0.008\right)$ and late $\left(6.87 \mathrm{~cm}^{3}\right.$ vs $\left.1.45 \mathrm{~cm}^{3}, p=0.008\right)$ volumes. Accumulated volume was not significantly greater in patients with leak recurrence $\left(0.58 \mathrm{~cm}^{3}\right.$ vs $\left.0.22 \mathrm{~cm}^{3}, p=0.07\right)$. Early, late, and accumulated volumes were significantly correlated with postoperative hospital stay as well as duration of postoperative lumbar drain placement ( $p<0.05$ for all measures).

CONCLUSIONS High-resolution CUBE T2 MRI, coupled with precise volumetric segmentation and subtraction of sinonasal hyperintensity, not only demonstrated predictive value in differentiating low- and high-flow CSF leaks, but also correlated with postoperative complications such as leak recurrence. These findings may be useful in the clinical workup and neurosurgical management of patients with skull base CSF leaks.

https://thejns.org/doi/abs/10.3171/2019.10.JNS192500

KEYWORDS cerebrospinal fluid leakage; flow rate; dural repair; primary spontaneous CSF leak; craniomaxillary trauma; endoscopic surgery; anterior skull base defect 
$\mathrm{C}$ SF leakage is a serious condition resulting from a defect in the arachnoid and dura mater and breach of the bony skull base contour, which together ordinarily prevent CSF from escaping the cranial vault. Potentially fatal complications can arise from CSF leakage, including meningitis, which occurs in $10 \%-25 \%$ of patients..$^{20,27,35} \mathrm{CSF}$ leakage has traditionally been classified as either traumatic or spontaneous. ${ }^{24} \mathrm{CSF}$ leakage resulting from traumatic cranial fracture has been reported in as high as $30 \%$ of cases, and craniomaxillary trauma accounts for approximately $80 \%$ of all CSF rhinorrhea cases. ${ }^{35}$ Another common etiology for anterior skull base leak results from thinning and erosion in idiopathic intracranial hypertension, a condition that typically occurs in middle-aged women with a body mass index (BMI) of above $30 \mathrm{~kg} / \mathrm{m}^{2}{ }^{6,23,28}$ Other etiologies include neoplasm, hydrocephalus, and congenital abnormalities. ${ }^{4,20,23}$ Whatever the cause, bony erosion at the skull base and subsequent tear in the arachnoid and dura permits escape of CSF between the CNS and compartments of the skull base, such as the nasal cavity and paranasal sinuses.

The goals of surgery for both traumatic and primary spontaneous CSF leaks are to seal the cranial defect and to separate the cranial vault from the sinonasal tract, while preserving ocular and cranial nerve function. Advancements in endoscopic transnasal sinus surgery have permitted high rates of successful CSF leak repair while minimizing the need for transcranial approaches to the anterior skull base. ${ }^{3,7,14}$ Despite many advances in the field of endoscopic surgery, the optimal diagnosis and management of anterior skull base CSF remains a clinical challenge. Disease features such as degree of CSF flow rate can complicate and compromise efficacy of surgical repair, with high-flow leaks increasing the likelihood of numerous adverse outcomes including postoperative leaks..$^{19}$

Prior studies have shown the importance of distinguishing high-flow from low-flow CSF leaks before surgery; ${ }^{21}$ however, current clinical tools are limited for preoperatively predicting objective flow rate. An imaging biomarker for quantifying CSF leak flow rate may prove useful for surgical planning, ${ }^{29}$ flap repair choice, ${ }^{15,19,25}$ indication for CSF diversion, $,, 7,14$ and predicting the likelihood of postoperative leak recurrence. ${ }^{14,15}$ The present study examines subtraction of fluid intensity volumes between early and late MRI acquisitions to quantify CSF flow rate noninvasively. We hypothesize that calculated volume differences correlate with the rate of CSF leakage clinically. Results from the present study may be useful in the preoperative workup and neurosurgical management for patients with primary spontaneous CSF leaks.

\section{Methods}

\section{Participant Selection}

Approval for this study was obtained from the local institutional review board. A total of 135 patients with suspected CSF leaks underwent MRI examination between November 2010 and May 2019. Inclusion criteria for the present study included traumatic or primary spontaneous anterior skull base CSF leaks. Of the cohort of 135 pa- tients, 90 were excluded from the study for the following reasons: prior sinus surgery, nonanterior skull base leak, and iatrogenic etiology. The remaining 45 patients met inclusion criteria for this study and were included in the analysis.

\section{Imaging Protocol}

The 3-T MRI was performed using a modified clinical sinus MRI protocol. CUBE T2 (the vendor trade name for volumetric extended echo train T2) sequences were obtained as the first scan following the localizer and cloned to repeat as the last sequence before scan conclusion. These early and late volumetric $\mathrm{T} 2$ sequences were thus obtained approximately 45 minutes apart. Additional clinical sequences obtained as standard of care included sagittal and axial T1; coronal single-shot T2; and axial T2, FLAIR, gradient echo and diffusion sequences, and postgadolinium volumetric $\mathrm{T} 1$ sequences obtained using a standard clinical weight-based dosing algorithm. MR images were examined by an experienced neuroradiologist who was blinded to intraoperative flow rate status, in order to determine whether there was evidence of CSF leakage.

\section{Image Analysis}

The two volumetric extended echo train sequences were transferred to a dedicated workstation for image analysis. Using a semiautomated method in 3D Slicer (http://www.slicer.org), the volume of CSF intensity was measured using a seed-based region-growing algorithm that included voxels greater than $50 \%$ of the maximum CSF signal intensity of the lateral ventricles. ${ }^{12}$ Volumetric segmentations were generated for both early and late acquisitions, with subtraction of early from late yielding the volume of interval CSF accumulation (Fig. 1). The semiautomated volumetric segmentation method used in this study is shown in Fig. 2.

\section{Clinical Data}

Disease features and demographic data were obtained from electronic medical records, intraoperative observations, laboratory testing, and radiological examinations. Definitive evidence of CSF leakage included a positive $\beta$-transferrin result, obvious CSF pooling in the sinonasal cavities, or intraoperative observation. Determination of the CSF leak flow rate was performed intraoperatively after exposure of the leak and after identification of its exact anatomical location. Criteria described by Esposito et al. were used to designate low-flow (small "weeping" leak, confirmed by Valsalva maneuver, without obvious or with only small diaphragmatic defect) and high-flow (moderate CSF leak, with obvious diaphragmatic defect) CSF leaks. ${ }^{11}$

\section{Statistical Analysis}

Statistical database software (JMP Pro version 14) was used for statistical analysis. Variables were assessed for normality by using the Shapiro-Wilk test. T-tests and Wilcoxon tests were used for continuous variables, as appropriate, and linear regression analyses were performed. Data were standardized and nominal logistic regression 

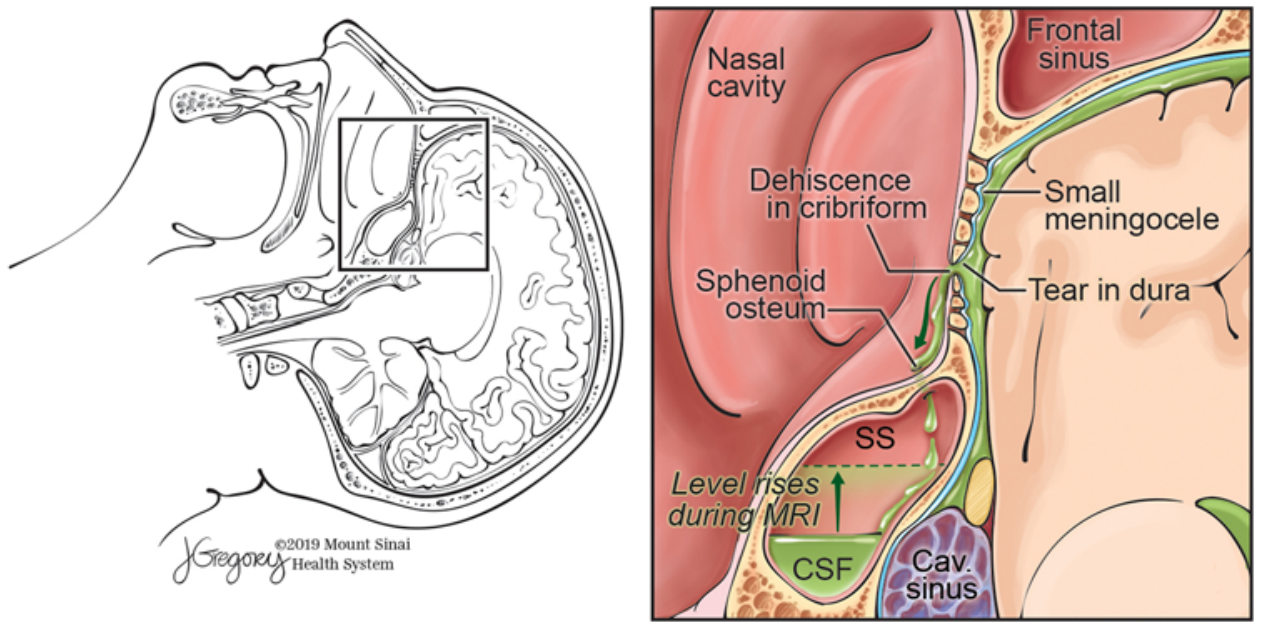

FIG. 1. Schematic depiction of the pooling mechanism in this study. The image on the left shows the location of details in the image on the right. Dural tear along with cribriform dehiscence allows CSF to trickle into the sinonasal cavity. Middle and posterior cribriform leaks tend to pool in the sphenoid sinus (SS), which is measurable by the change in fluid intensity during the course of the imaging session. More anterior leaks, which can ultimately bypass the sphenoid, may instead also show fluid coating mucosa and possible layering in the nasopharynx. Cav. = cavernous. Illustrator: Jill K. Gregory. Printed with permission from @2019 Mount Sinai Health System. Figure is available in color online only.

was used to predict high-flow leak and leak recurrence. A $\mathrm{p}$ value $\leq 0.05$ was used to denote significance.

\section{Results}

A total of 45 patients ( 28 females, 17 males, average age 49.9 years) met inclusion criteria. Out of this cohort,
25 patients (16 females, 9 males, average age 51.9 years) showed definitive evidence of anterior skull base CSF leakage. The most prevalent comorbidities in the patients with definitive CSF leaks were hypertension $(16 / 25,64 \%)$, obesity $(16 / 25,64 \%)$, and diabetes mellitus $(7 / 25,28 \%)$. The mean BMI was $34.2 \mathrm{~kg} / \mathrm{m}^{2}$ (SD $8.4 \mathrm{~kg} / \mathrm{m}^{2}$ ). Presenting symptoms are shown in Table 1 . Of the patients with
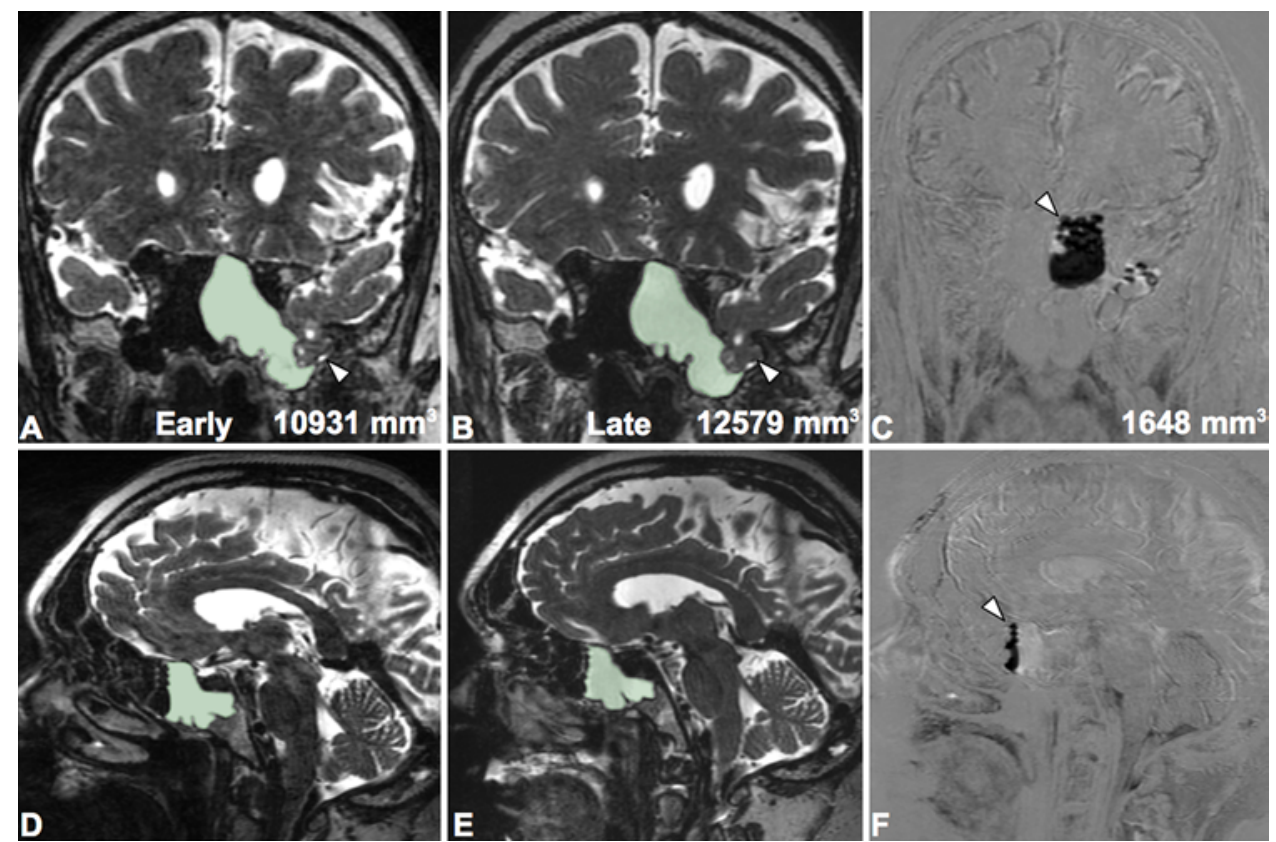

FIG. 2. A 78-year-old man presented with 1 month of headache and left-sided rhinorrhea resulting from a high-flow leak with pooling from a left sphenoid encephalocele (white arrowheads, $A$ and B). Panels $\mathbf{A}$ and $\mathbf{B}$ are $16 \mathrm{~mm}$ anterior and panel $\mathbf{C}$ is $20 \mathrm{~mm}$ anterior to mid-dorsum, whereas panels D-F depict a plane $5 \mathrm{~mm}$ left of midline. Image subtractions of early minus late T2 reveal new low signal (hypointensity denoted by white arrowheads), representing considerable new layering signal accumulation between $s c a n s(A, D=$ early T2; B, E = late T2; C, F = subtraction). This is consistent with CSF pooling. Figure is available in color online only. 
TABLE 1. Presenting symptoms of the patients included in the present study

\begin{tabular}{lcc}
\hline Presenting Symptom & $\begin{array}{c}\text { No. (\%) for } \\
\text { Entire Cohort, } \\
\mathrm{n}=45\end{array}$ & $\begin{array}{c}\text { No. (\%) of Patients } \\
\text { w/ Confirmed CSF } \\
\text { Leaks, } \mathrm{n}=25\end{array}$ \\
\hline Headache & $30(66.7)$ & $17(68)$ \\
\hline Rhinorrhea & $35(77.8)$ & $22(88)$ \\
\hline Tinnitus/hearing loss & $7(15.6)$ & $5(20)$ \\
\hline Vision change & $9(20)$ & $5(20)$ \\
\hline Nausea & $10(22.2)$ & $6(24)$ \\
\hline Vomiting & $2(4.4)$ & $0(0)$ \\
\hline Dizziness & $6(13.3)$ & $4(16)$ \\
\hline Salty taste & $7(15.6)$ & $5(20)$ \\
\hline Fatigue & $7(15.6)$ & $2(8)$ \\
\hline Vertigo & $3(6.7)$ & $0(0)$ \\
\hline Imbalance & $2(4.4)$ & $1(4)$ \\
\hline Facial pain/pressure & $8(17.8)$ & $3(12)$ \\
\hline Seizure & $1(2.2)$ & $0(0)$ \\
\hline Loss of consciousness & $1(2.2)$ & $0(0)$ \\
\hline Sinonasal congestion & $9(20)$ & $6(24)$ \\
\hline Neck pain/stiffness & $6(13.3)$ & $4(16)$ \\
\hline Photophobia & $4(8.9)$ & $2(8)$ \\
\hline Epistaxis & $1(2.2)$ & $2(8)$ \\
\hline Meningitis & $4(8.9)$ & $4(16)$ \\
\hline Hyposmia/anosmia & $3(6.7)$ & $1(4)$ \\
\hline
\end{tabular}

definitive leaks, 23/25 (92\%) showed CSF pooling in the sinonasal compartments on the extended echo train T2 sequence, and 22/25 (88\%) patients with confirmed CSF leaks underwent endoscopic repair surgery. Demographic data, disease features, and treatment outcomes are shown in Table 2.

\section{CSF Volumes and Surgical Results}

High-flow leaks were identified intraoperatively in $10 / 22$ endoscopically treated patients $(45.5 \%)$, whereas low-flow leaks were seen in 12/22 (54.5\%). The mean volume of fluid intensity on early scans among patients with low-flow leaks was $982.0 \pm 1527.8 \mathrm{~mm}^{3}$ (mean $\pm \mathrm{SD}$ ), significantly lower than that of high-flow leaks (4057.4 \pm $\left.4325.5 \mathrm{~mm}^{3}\right)(\mathrm{p}=0.04)$. The average volume of fluid intensity on late scans among patients with low-flow leaks was $1095.6 \pm 1601.7 \mathrm{~mm}^{3}$, significantly lower than in those with high-flow leaks $\left(4584 \pm 4817.5 \mathrm{~mm}^{3}\right)(\mathrm{p}=0.04)$. The mean accumulation in CSF volume between the early and late acquisitions for patients with low-flow leaks was 113.6 $\pm 139.6 \mathrm{~mm}^{3}$, significantly lower than that of patients with high-flow leaks $\left(526.6 \pm 579.6 \mathrm{~mm}^{3}\right)(\mathrm{p}=0.01)$. Patients with constant rhinorrhea did not have significantly different early, late, or accumulated CSF volumes compared with patients who experienced intermittent rhinorrhea ( $\mathrm{p}$ $>0.05$ for all; Table 3). Volumetric measurement and image subtraction in patients with a high-flow and low-flow CSF leak are shown in Figs. 2 and 3, respectively.

Middle turbinate grafts were used in 20/22 endoscopic
TABLE 2. Demographic data, disease features, treatments, and outcomes for 25 patients with confirmed anterior skull base CSF leaks

\begin{tabular}{|c|c|}
\hline Characteristic & Value \\
\hline \multicolumn{2}{|l|}{ Demographics } \\
\hline Female & $16(64)$ \\
\hline Age in yrs, mean $\pm S D$ & $51.9 \pm 9.9$ \\
\hline BMI in $\mathrm{kg} / \mathrm{m}^{2}$, mean $\pm \mathrm{SD}$ & $34.2 \pm 8.4$ \\
\hline \multicolumn{2}{|l|}{ Disease features } \\
\hline Symptom duration in mos, mean \pm SD & $13.1 \pm 21.7$ \\
\hline Head/sinus trauma & $5(20)$ \\
\hline Left-sided defect & $13(52)$ \\
\hline Right-sided defect & $12(48)$ \\
\hline \multicolumn{2}{|l|}{ Defect location } \\
\hline Cribriform & $15(60)$ \\
\hline Fovea & $2(8)$ \\
\hline Sphenoid & $6(24)$ \\
\hline Ethmoid & $2(8)$ \\
\hline \multicolumn{2}{|l|}{ Treatment } \\
\hline Positive B-transferrin & $18(72)$ \\
\hline Cisternography & $2(8)$ \\
\hline \multicolumn{2}{|l|}{ Surgical features } \\
\hline Endoscopic surgery & $22(88)$ \\
\hline Fluorescein & $15(68.2)$ \\
\hline Lumbar drain & $17(77.3)$ \\
\hline VP shunt & $4(18.2)$ \\
\hline \multicolumn{2}{|l|}{ Graft material } \\
\hline Middle turbinate & $20(90.9)$ \\
\hline Mucoperiosteal/osteoperiosteal & $2(9.1)$ \\
\hline \multicolumn{2}{|l|}{ Surgical outcomes } \\
\hline Postop stay in days, mean \pm SD & $2.5 \pm 1.12$ \\
\hline Recurrence & $5(22.7)$ \\
\hline Time to recurrence in days, mean $\pm S D$ & $143.6 \pm 305.5$ \\
\hline Subsequent surgery & $4(18.2)$ \\
\hline Length of follow-up in days, mean $\pm S D$ & $377.9 \pm 487.5$ \\
\hline
\end{tabular}

Unless otherwise indicated, values are expressed as the number of patients $(\%)$.

(90.9\%) cases, and free mucoperiosteal/osteoperiosteal grafts were used in $2 / 22(9.1 \%)$. Perioperative lumbar drains were used in 17/22 (77.3\%) surgeries, and other CSF diversion methods such as ventriculoperitoneal (VP) shunts were used in 4/22 (18.2\%). The mean postoperative stay and duration of perioperative lumbar diversion were $2.5 \pm 1.12$ days and $2.21 \pm 1.03$ days, respectively.

There were no significant differences in or accumulated fluid volumes between spontaneous and traumatic CSF leaks ( $p>0.05$ for all).

\section{CSF Volumes and Treatment Outcomes}

Five of 22 endoscopically treated patients (22.7\%) exhibited recurrence of CSF leakage following surgery, with time to recurrence of $143.6 \pm 305.5$ days. Four patients 
TABLE 3. Early, late, and accumulated CSF leak volume based on flow properties and surgical outcomes

\begin{tabular}{|c|c|c|c|c|}
\hline Variable & No. of Patients & Early CSF Vol $\left(\mathrm{mm}^{3}\right)$ & Late CSF Vol $\left(\mathrm{mm}^{3}\right)$ & Accumulated CSF Vol $\left(\mathrm{mm}^{3}\right)$ \\
\hline \multicolumn{5}{|l|}{ Flow rate } \\
\hline Low flow & 12 & $982.0(1527.8)$ & $1095.6(1601.7)$ & $113.6(139.6)$ \\
\hline High flow & 10 & $4057.4(4325.5)$ & $4584(4817.5)$ & $526.6(579.6)$ \\
\hline$p$ value & & 0.04 & 0.04 & 0.01 \\
\hline \multicolumn{5}{|l|}{ Recurrence } \\
\hline Nonrecurrence & 17 & $1227.8(2582.9)$ & $1447.9(2952.4)$ & $220.1(392.1)$ \\
\hline Recurrence & 5 & $6296.9(3148)$ & $6874.4(3543.9)$ & $577.5(551.1)$ \\
\hline$p$ value & & 0.008 & 0.0008 & 0.07 \\
\hline \multicolumn{5}{|l|}{ Subsequent repair } \\
\hline No subsequent repair & 18 & $1699.0(3211.3)$ & $1934.6(3548.4)$ & $235.6(391.9)$ \\
\hline Subsequent repair & 4 & $5443.7(2854.8)$ & $6040.8(3378.3)$ & $597.1(613.4)$ \\
\hline$p$ value & & 0.03 & 0.03 & 0.12 \\
\hline \multicolumn{5}{|l|}{ Rhinorrhea flow } \\
\hline Intermittent rhinorrhea & 11 & $2471.1(3133.3)$ & $2717(3343.5)$ & $246(267)$ \\
\hline Constant rhinorrhea & 5 & $2013.4(2539.2)$ & $2227.2(2678.7)$ & $213.7(214.1)$ \\
\hline$p$ value & & $>0.05$ & $>0.05$ & $>0.05$ \\
\hline
\end{tabular}

Values are expressed as the mean (SD). Boldface type indicates statistical significance.

(18.2\%), 2 with low-flow and 2 with high-flow leaks (determined intraoperatively), required subsequent CSF leak revision surgery. Of the 5 patients with leak recurrence, 2 underwent postoperative MRI with the same imaging protocol. One patient had early, late, and accumulated fluid volumes of $2205.1 \mathrm{~mm}^{3}, 3453.86 \mathrm{~mm}^{3}$, and $1248.8 \mathrm{~mm}^{3}$, and the other patient had fluid volumes of $322.7 \mathrm{~mm}^{3}$, $1243.2 \mathrm{~mm}^{3}$, and $920.5 \mathrm{~mm}^{3}$, respectively.

The mean early fluid volume for the 5 patients who demonstrated postoperative CSF leak recurrence (6296.9 \pm $3148.0 \mathrm{~mm}^{3}$ ) was significantly greater than that of patients who did not experience postoperative leakage (1227.8 \pm $\left.2582.9 \mathrm{~mm}^{3}\right)(\mathrm{p}<0.01)$. The mean late fluid volume for patients who demonstrated postoperative CSF leak recurrence $\left(6874.4 \pm 3543.9 \mathrm{~mm}^{3}\right)$ was significantly greater than that of patients who did not experience postoperative leakage $\left(1447.9 \pm 2952.4 \mathrm{~mm}^{3}\right)(\mathrm{p}<0.01)$. The mean accumulated CSF volume for the 5 patients who demonstrated postoperative CSF leak recurrence $\left(577.5 \pm 551.1 \mathrm{~mm}^{3}\right)$ was not significantly different than that of patients without postoperative leakage $\left(220.1 \pm 392.1 \mathrm{~mm}^{3}\right)(\mathrm{p}=0.07)$.

The mean early fluid intensity for the 4 patients who required subsequent surgical CSF leak repair (5443.7 \pm $2854.8 \mathrm{~mm}^{3}$ ) was significantly greater than that of patients who did not require secondary surgery $(1699.0 \pm 3211.3$ $\left.\mathrm{mm}^{3}\right)(\mathrm{p}=0.03)$. The mean late fluid intensity of patients who underwent subsequent surgical CSF leak repair $\left(6040.8 \pm 3378.3 \mathrm{~mm}^{3}\right)$ was significantly greater than that of patients who did not require secondary surgery (1934.6 $\left.\pm 3548.4 \mathrm{~mm}^{3}\right)(\mathrm{p}=0.03)$. The mean accumulated CSF volume for patients who underwent subsequent surgical CSF leak repair $\left(597.1 \pm 613.4 \mathrm{~mm}^{3}\right)$ was not significantly different from that of patients who did not require secondary surgery $\left(235.6 \pm 391.9 \mathrm{~mm}^{3}\right)(\mathrm{p}=0.12)$. These results are shown in Table 3.

Accumulated CSF volume was the most significant fac- tor for predicting flow rate. For every 1-SD increase in volume difference, the odds of a high-flow leak increased 11 -fold (95\% CI 1.5, 238.8) (p = 0.008). Early CSF volume was the most significant predictor for postoperative leak recurrence, so for each SD increase in early volume, the odds of recurrence increased by 4.6 -fold $(95 \%$ CI 1.5 , 22.0) $(\mathrm{p}=0.005)$.

CSF leak volume positively correlated with postoperative hospital stay for early $(r=0.51, p=0.03)$, late $(r=0.53$, $\mathrm{p}=0.02)$, and accumulated volumes $(\mathrm{r}=0.57, \mathrm{p}=0.01)$. CSF leak volume also positively correlated with duration of postoperative lumbar drainage for early $(\mathrm{r}=0.70, \mathrm{p}<$ $0.001)$, late $(\mathrm{r}=0.70, \mathrm{p}<0.001)$, and accumulated volumes $(\mathrm{r}=0.63, \mathrm{p}=0.004)$ (Fig. 4). BMI was not significantly correlated with early $(r=-0.26)$, late $(r=-0.23)$, or accumulated $(r=-0.09)$ CSF volumes ( $p>0.05$ for all).

\section{Discussion}

The present study uses a semiautomated region-growing approach to measure pooled CSF volume in the sinonasal tract and volume subtraction between early and late high-resolution T2-weighted extended echo train T2 acquisitions as a method of characterizing CSF flow rate in anterior skull base rhinorrhea. We present evidence suggesting that increased early, late, and accumulated sinonasal fluid volumes are associated with high-flow leaks. High early and late volumes may be useful in prediction of the likelihood of postoperative leak recurrence and need for subsequent repair. The methodology and results presented here may be useful in the neurosurgical workup and management of patients with anterior skull base CSF leaks.

Critical factors in the management of anterior skull base CSF leaks include defect location, intraoperative flow rate, type of reconstruction materials, and adjuvant radia- 

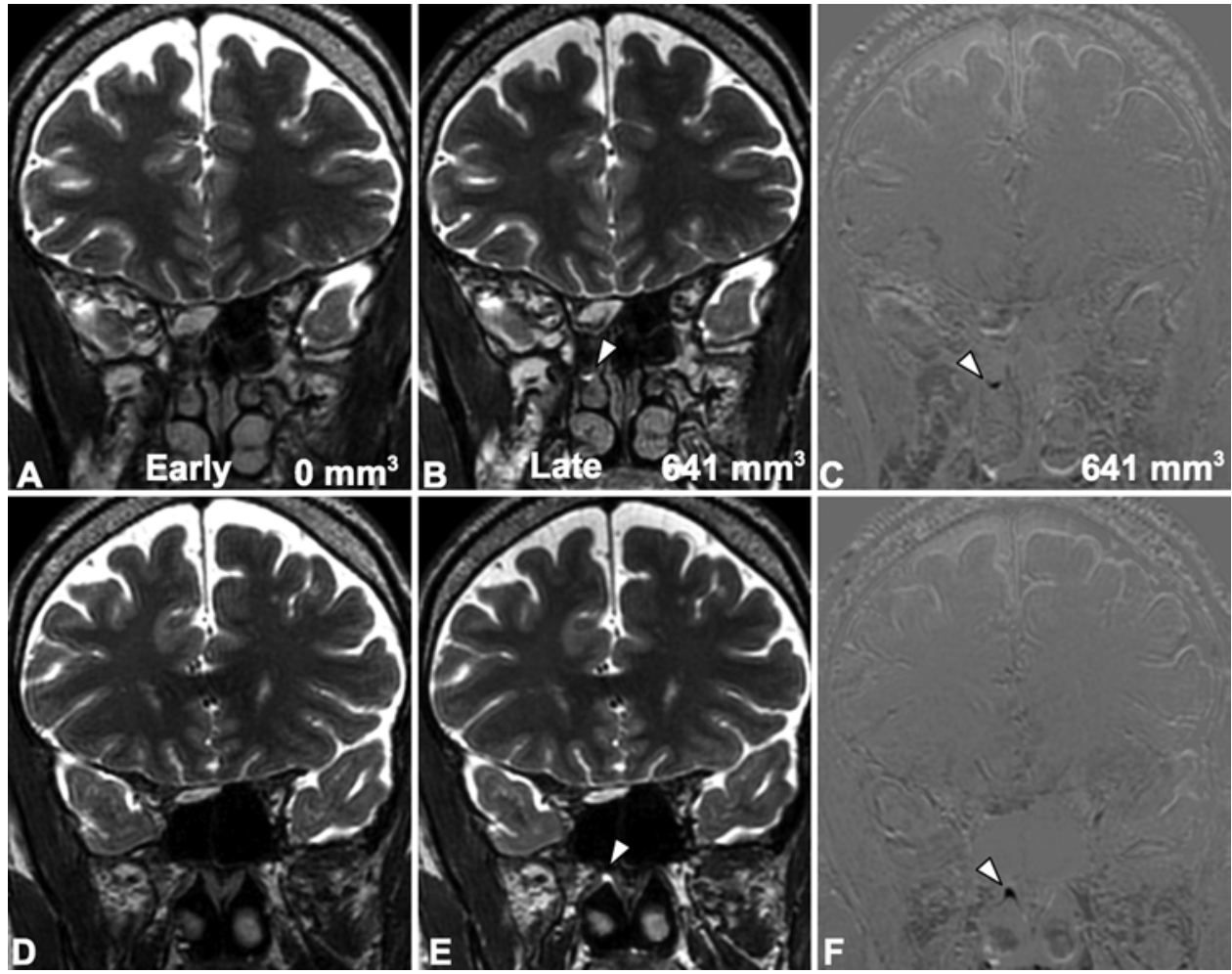

FIG. 3. A 43-year-old man presented with intermittent right-sided rhinorrhea and nasal congestion resulting from a low-flow CSF leak from a right cribriform meningoencephalocele. Panels $A-C$ are $27 \mathrm{~mm}$ anterior to mid-dorsum, and panels $\mathbf{D}-\mathrm{F}$ demonstrate a plane $18 \mathrm{~mm}$ anterior to mid-dorsum sellae. Panels $A-C$ and $D-F$ use the same timing/subtraction conventions as in Fig. 2. No adherent CSF signal is observed in the early acquisitions ( $A$ and $D$ ); however, new fluid intensity (denoted by white arrowheads) appears in the late acquisitions ( $B$ and $E$ ). Image subtractions ( $C$ and $F$ ) of early minus late $T 2$ show corresponding low signal, which in this subtraction methodology reflects new T2 hyperintensity compatible with fluid (denoted by white arrowheads).

tion therapy., ${ }^{4,61}$ The present study examines noninvasive preoperative imaging as a method to quantify CSF flow rate. Prior studies have proposed novel methods of localizing the defect site of high-flow CSF leaks, which can be obscured by leaked intrathecal contrast material that appears in multiple levels during CT as a result of brisk flow. Luetmer and Mokri used dynamic CT myelography and reported increased visibility of the origin site in patients with high-flow spontaneous CSF leaks; however, to our knowledge there has not been a study that examines pre- operative quantification of CSF flow rate. ${ }^{18}$ The objective of the present study was to examine the utility of CSF leak volume subtraction in which high-resolution early and late T2 extended echo train MRI acquisitions were used in determining the flow rate of CSF leaks. Our results suggest that this technique may be a sensitive method of predicting flow rate, which may be useful in the preoperative workup, prognosis, and management of patients with anterior skull base CSF leaks.

CSF leaks are typically classified as low- or high-flow

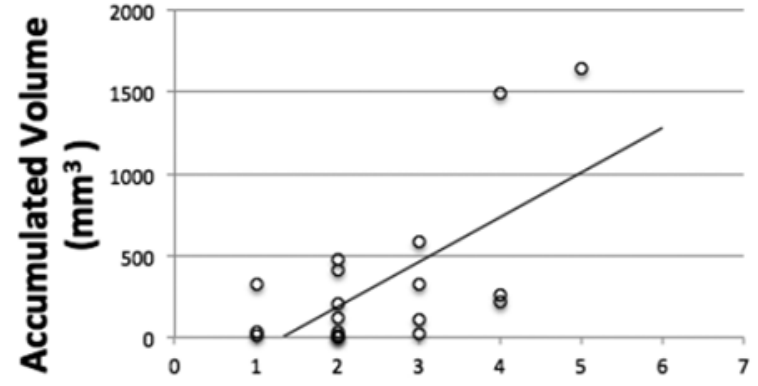

A

Hospital Stay (days)

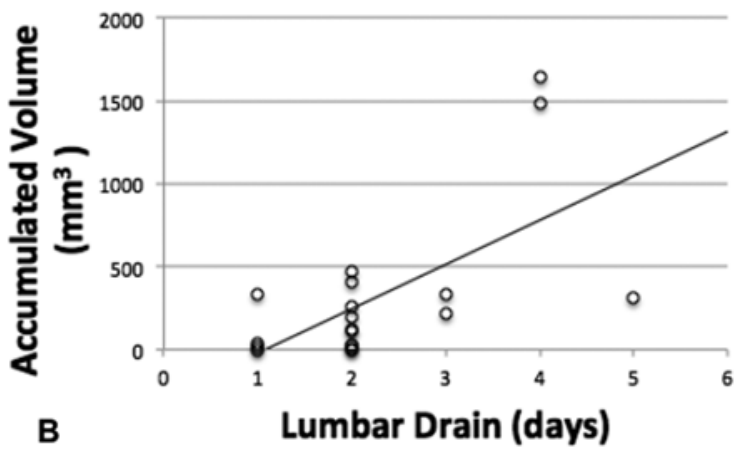

FIG. 4. Graphs showing Pearson correlations between accumulated CSF leak volume against both duration of postoperative hospital stay $(r=0.57, p=0.01, A)$ and duration of postoperative lumbar drain CSF diversion $(r=0.63, p=0.004, B)$. 
intraoperatively. Accurate preoperative prediction of the CSF leak flow rate is valuable, because flow conditions through the skull base defect can impact therapeutic procedures. There are currently no widely accepted quantitative thresholds defining high versus low flow rates, but it is believed that high-flow leaks result from direct CSF transmission between the dural defect and paranasal sinuses. ${ }^{31}$ High-flow leaks rarely close naturally and typically require surgical repair to seal the defect. ${ }^{25,28}$ Additional factors suggesting possible benefit from repair surgery include tension pneumocephalus and large dural defects. ${ }^{35}$

For low-flow leaks, conservative measures such as bed rest, head elevation, and CSF diversion may be effective in allowing less significant dural defects to close spontaneously. ${ }^{22,35}$ When surgical intervention is necessary, low-flow CSF leaks often can be controlled well with endoscopic surgery. Free fat grafts, acellular graft materials such as Alloderm, and overlaid synthetic material have been used to successfully repair low-flow leaks. ${ }^{19,30,33}$ The dura mater can also be sutured endoscopically to close low-pressure dural defects. ${ }^{8}$ Advantages of endoscopic surgery are the reduced need for skeletal disassembly and brain retraction, and shorter recovery time. ${ }^{16}$

When broader exposure is necessary, CSF leaks can also be corrected with transcranial surgery, which often includes a bifrontal craniotomy permitting more complete access to the defect. A transcranial approach to the anterior skull base may be preferred over endoscopic surgery to address frontal sinus damage, complex skull base traumatic defects, and accompanying cranial nerve damage. ${ }^{16}$ In addition to these factors, multifocal and high-volume CSF leaks can also be indications for transcranial surgery, because these leaks may not be amenable to endoscopic repair, particularly when vascularized pericranial flaps are required..$^{2,22,33}$ Vascularized flap repair (including turbinate and nasoseptal flaps) results in significantly greater success rates for high-flow CSF leaks compared with nonvascularized techniques (in one series the success rate was $94 \%$ vs $82 \%){ }^{10,13,31} \mathrm{Hu}$ et al. discussed the importance of harvesting a vascularized pedicle nasoseptal flap early in the course of surgery for patients with expected high-flow leaks..$^{15}$ A Hadad-Bassagasteguy flap may be used with high-flow leaks, although Luginbuhl et al. also describe a button graft for endoscopic repair of high-flow CSF leaks. ${ }^{19}$ It is worth emphasizing that transcranial approaches are associated with multiple intraoperative risks including cerebral hemorrhage, frontal lobe damage from prolonged brain retraction, and anosmia, as well as increased risk of recurrence and lengthened postoperative stay. ${ }^{16}$ Therefore, transcranial surgery should be reserved for patients with high-flow CSF leaks that cannot be adequately repaired endoscopically.

Intraoperative procedures may vary depending on CSF flow rate, and preoperative knowledge of flow conditions may be useful in surgical decision-making. For patients with a high likelihood of a high-flow leak, a nasoseptal flap is harvested early during the repair. ${ }^{25}$ Additionally, prophylactic intraoperative lumbar drains are commonly used in the setting of high-flow CSF leaks to relieve pressure postoperatively and to reduce the pressure gradient across the repaired surface. ${ }^{14,31}$ Selective lumbar drains may be useful in patients with high-flow or persistent leaks. ${ }^{5,7,14}$ Lumbar drains are typically left in place for 24 to 120 hours after surgery to regulate CSF pressure and preserve graft adhesion to the skull base defect. ${ }^{14}$ However, use of prophylactic lumbar drains to prevent postoperative CSF leaks is controversial and varies across institutions. CSF diversion should only be pursued when needed because this procedure may also lead to intracranial hypotension and pneumocephalus. ${ }^{4}$ Indeed, some investigators have reported excellent surgical outcomes using this method, ${ }^{36}$ whereas others have shown that perioperative CSF lumbar drains are associated with a higher risk of meningitis and ventriculitis. ${ }^{1,9}$ However, the value of perioperative CSF diversion for various CSF flow rates is still poorly understood, and future studies are needed to better elucidate clinical indications for this procedure..$^{31}$

There is also a paucity of objective criteria defining which cases might require VP shunts, which can be used in conjunction with direct defect repair or as a stand-alone intervention. VP shunts are sometimes used when patients report pressure headaches or visual changes. Some institutions reserve VP shunts for patients with very elevated intracranial pressure, ${ }^{17}$ whereas others reserve this method for repeat skull base repair surgery. ${ }^{25} \mathrm{VP}$ shunts in the setting of high-flow CSF leaks may increase the risk of pneumocephalus, presumably by drawing air into a more substantial defect than might be found in a low-flow leak. ${ }^{32}$ Accordingly, Spetzler and Wilson have described their practice in which VP shunts are used for low-flow fistulas and direct repair is used for high-flow fistulas..$^{32}$ Nevertheless, there does not appear to be a clear consensus for indications for VP shunt diversion.

There remains considerable uncertainty with regard to which patients benefit most from CSF diversion, the diversion method to be used, and the duration of diversion. ${ }^{35} \mathrm{VP}$ shunts, like lumbar drains, are associated with potentially life-threatening complications including meningitis and shunt failure. This procedure ought to be used in patients for whom clinical benefit may be experienced. ${ }^{17}$ Our CSF leak flow rate, measured by preoperative MRI, may prove to be useful in identifying candidates for VP shunts. Future work will be necessary to determine the utility of this method in planning CSF diversion.

Preoperative prediction of CSF flow rate may help guide selection of repair materials beyond graft choice and use of CSF diversion. Applying self-setting hydroxyapatite bone cement can repair low-flow CSF leaks that are due to a sphenoid sinus defect. However, this method is contraindicated in the setting of high-flow leaks, because the cement can be washed away by the fast-moving CSF before it sets. ${ }^{4,14,34}$ Additionally, high pulsatility of CSF exiting from high-flow leak defects can also result in microfracturing of cement, further limiting its utility in treating high-flow leaks. ${ }^{14,34}$

In addition to guiding intraoperative decision-making, our results indicate that volumetric analysis of CSF leaks can also be used to predict the likelihood of postoperative leak recurrence and need for subsequent repair. Despite the low rate of recurrence in this study, patients with postoperative leak recurrence as well as those who required subsequent repair surgery demonstrated significantly 
greater early and late CSF volumes. Currently, prognostic factors predicting the likelihood of recurrent CSF leaks are limited, although location of the skull base defect has been shown to be a significant indicator, with frontal sinus repairs exhibiting the highest failure rates. ${ }^{26}$ Volumetric analysis of CSF leaks may serve as an imaging biomarker to identify patients with an increased likelihood of postoperative leak recurrence and may be useful in the management and early risk stratification of patients with anterior skull base CSF leaks.

\section{Limitations and Future Directions}

Like CSF, skull base mucosa appears hyperintense on T2 CUBE imaging. The segmentation method in this study is likely to include small areas of mucosa in the CSF volume measurements. In order to limit this source of error, half of the signal intensity of the CSF in the lateral ventricles was selected as an objective lower signal intensity threshold. The signal intensity of the mucosa at the skull base did not reach this threshold in most cases; however, areas of mucosal thickening may have resulted in some mucosa being included in fluid volume calculations and was a potential source of error in this study. However, because the same technique was used for the early and late scan measurement, the difference calculation should cancel such measurement error in most cases.

In an effort to study a homogeneous cohort of patients with primary spontaneous and traumatic CSF leaks, patients with iatrogenic leaks or prior sinonasal surgery were excluded from the present study. CSF leaks resulting from surgery are common clinical scenarios, and future work will investigate flow rate patterns in these patients. Additionally, the small number of patients who exhibited negative postoperative outcomes precluded correlation between CSF leak volumes and time to leak recurrence or subsequent repair surgery. Future work examining the relationship between CSF leak volume and interval to repair failure is warranted.

Finally, in this study, CSF leaks were designated as high or low flow based on intraoperative observation. Whereas current flow rate classifications are typically binary in nature, CSF flow represents a continuum. We believe that the methodology presented here provides data that may be useful in the quantitative evaluation of CSF leak flow rate, which could allow for more accurate prediction of clinical outcomes including intraoperative conditions and postoperative recovery.

\section{Conclusions}

The present study demonstrates the radiological metrics that may prove useful in characterizing anterior skull base CSF leak flow rate, and could ultimately aid in predicting negative clinical outcomes such as postoperative leak recurrence. Prior studies have shown that various factors including surgical planning, repair materials, and prognosis can be affected by CSF leak flow rate. Early identification of patients with high-flow leaks may help determine treatment and better stratify risk. Preoperative knowledge of CSF flow conditions may be valuable in the diagnosis and management of patients with CSF leaks. Future studies to evaluate nonanterior skull base and iatrogenic CSF leaks may provide additional clinical value.

\section{References}

1. Ahmed OH, Marcus S, Tauber JR, Wang B, Fang Y, Lebowitz RA: Efficacy of perioperative lumbar drainage following endonasal endoscopic cerebrospinal fluid leak repair. Otolaryngol Head Neck Surg 156:52-60, 2017

2. Barger J, Siow M, Kader M, Phillips K, Fatterpekar G, Kleinberg D, et al: The posterior nasoseptal flap: a novel technique for closure after endoscopic transsphenoidal resection of pituitary adenomas. Surg Neurol Int 9:32, 2018

3. Bernal-Sprekelsen M, Alobid I, Mullol J, Trobat F, TomásBarberán M: Closure of cerebrospinal fluid leaks prevents ascending bacterial meningitis. Rhinology 43:277-281, 2005

4. Carrau RL, Snyderman CH, Kassam AB: The management of cerebrospinal fluid leaks in patients at risk for high-pressure hydrocephalus. Laryngoscope 115:205-212, 2005

5. Casiano RR, Jassir D: Endoscopic cerebrospinal fluid rhinorrhea repair: is a lumbar drain necessary? Otolaryngol Head Neck Surg 121:745-750, 1999

6. Chaaban MR, Illing E, Riley KO, Woodworth BA: Spontaneous cerebrospinal fluid leak repair: a five-year prospective evaluation. Laryngoscope 124:70-75, 2014

7. Conger A, Zhao F, Wang X, Eisenberg A, Griffiths C, Esposito F, et al: Evolution of the graded repair of CSF leaks and skull base defects in endonasal endoscopic tumor surgery: trends in repair failure and meningitis rates in 509 patients. J Neurosurg 130:861-875, 2018

8. Cukurova I, Cetinkaya EA, Aslan IB, Ozkul D: Endonasal endoscopic repair of ethmoid roof cerebrospinal fluid fistula by suturing the dura. Acta Neurochir (Wien) 150:897-900, 2008

9. D'Anza B, Tien D, Stokken JK, Recinos PF, Woodard TR, Sindwani R: Role of lumbar drains in contemporary endonasal skull base surgery: meta-analysis and systematic review. Am J Rhinol Allergy 30:430-435, 2016

10. Ecin G, Oner AY, Tokgoz N, Ucar M, Aykol S, Tali T: T2weighted vs. intrathecal contrast-enhanced MR cisternography in the evaluation of CSF rhinorrhea. Acta Radiol 54:698-701, 2013

11. Esposito F, Dusick JR, Fatemi N, Kelly DF: Graded repair of cranial base defects and cerebrospinal fluid leaks in transsphenoidal surgery. Oper Neurosurg (Hagerstown) 60 (4 Suppl 2):295-304, 2007

12. Fedorov A, Beichel R, Kalpathy-Cramer J, Finet J, FillionRobin JC, Pujol S, et al: 3D Slicer as an image computing platform for the Quantitative Imaging Network. Magn Reson Imaging 30:1323-1341, 2012

13. Harvey RJ, Parmar P, Sacks R, Zanation AM: Endoscopic skull base reconstruction of large dural defects: a systematic review of published evidence. Laryngoscope 122:452-459, 2012

14. Hegazy HM, Carrau RL, Snyderman CH, Kassam A, Zweig J: Transnasal endoscopic repair of cerebrospinal fluid rhinorrhea: a meta-analysis. Laryngoscope 110:1166-1172, 2000

15. Hu F, Gu Y, Zhang X, Xie T, Yu Y, Sun C, et al: Combined use of a gasket seal closure and a vascularized pedicle nasoseptal flap multilayered reconstruction technique for highflow cerebrospinal fluid leaks after endonasal endoscopic skull base surgery. World Neurosurg 83:181-187, 2015

16. Liu P, Wu S, Li Z, Wang B: Surgical strategy for cerebrospinal fluid rhinorrhea repair. Neurosurgery 66 (6 Suppl Operative):281-286, 2010

17. Lobo BC, Baumanis MM, Nelson RF: Surgical repair of spontaneous cerebrospinal fluid (CSF) leaks: a systematic review. Laryngoscope Investig Otolaryngol 2:215-224, 2017 
18. Luetmer PH, Mokri B: Dynamic CT myelography: a technique for localizing high-flow spinal cerebrospinal fluid leaks. AJNR Am J Neuroradiol 24:1711-1714, 2003

19. Luginbuhl AJ, Campbell PG, Evans J, Rosen M: Endoscopic repair of high-flow cranial base defects using a bilayer button. Laryngoscope 120:876-880, 2010

20. Maher CO, Meyer FB, Mokri B: Surgical treatment of spontaneous spinal cerebrospinal fluid leaks. Neurosurg Focus 9(1):e7, 2000

21. McCoul ED, Anand VK, Singh A, Nyquist GG, Schaberg MR, Schwartz TH: Long-term effectiveness of a reconstructive protocol using the nasoseptal flap after endoscopic skull base surgery. World Neurosurg 81:136-143, 2014

22. Meier JC, Bleier BS: Novel techniques and the future of skull base reconstruction. Adv Otorhinolaryngol 74:174-183, 2013

23. O'Connell JE: Primary spontaneous cerebrospinal fluid rhinorrhoea. J Neurol Neurosurg Psychiatry 27:241-246, 1964

24. Ommaya AK: Cerebrospinal fluid rhinorrhea. Neurology 14:106-113, 1964

25. Patel KS, Komotar RJ, Szentirmai O, Moussazadeh N, Raper DM, Starke RM, et al: Case-specific protocol to reduce cerebrospinal fluid leakage after endonasal endoscopic surgery. J Neurosurg 119:661-668, 2013

26. Purkey MT, Woodworth BA, Hahn S, Palmer JN, Chiu AG: Endoscopic repair of supraorbital ethmoid cerebrospinal fluid leaks. ORL J Otorhinolaryngol Relat Spec 71:93-98, 2009

27. Schievink WI: Spontaneous spinal cerebrospinal fluid leaks and intracranial hypotension. JAMA 295:2286-2296, 2006

28. Schlosser RJ, Wilensky EM, Grady MS, Bolger WE: Elevated intracranial pressures in spontaneous cerebrospinal fluid leaks. Am J Rhinol 17:191-195, 2003

29. Schlosser RJ, Wilensky EM, Grady MS, Palmer JN, Kennedy DW, Bolger WE: Cerebrospinal fluid pressure monitoring after repair of cerebrospinal fluid leaks. Otolaryngol Head Neck Surg 130:443-448, 2004

30. Senior BA, Jafri K, Benninger M: Safety and efficacy of endoscopic repair of CSF leaks and encephaloceles: a survey of the members of the American Rhinologic Society. Am J Rhinol 15:21-25, 2001

31. Soudry E, Turner JH, Nayak JV, Hwang PH: Endoscopic reconstruction of surgically created skull base defects: a systematic review. Otolaryngol Head Neck Surg 150:730-738, 2014
32. Spetzler RF, Wilson CB: Management of recurrent CSF rhinorrhea of the middle and posterior fossa. J Neurosurg 49:393-397, 1978

33. Thorp BD, Sreenath SB, Ebert CS, Zanation AM: Endoscopic skull base reconstruction: a review and clinical case series of 152 vascularized flaps used for surgical skull base defects in the setting of intraoperative cerebrospinal fluid leak. Neurosurg Focus 37(4):E4, 2014

34. Yadav YR, Parihar V, Janakiram N, Pande S, Bajaj J, Namdev H: Endoscopic management of cerebrospinal fluid rhinorrhea. Asian J Neurosurg 11:183-193, 2016

35. Ziu M, Savage JG, Jimenez DF: Diagnosis and treatment of cerebrospinal fluid rhinorrhea following accidental traumatic anterior skull base fractures. Neurosurg Focus 32(6):E3, 2012

36. Zwagerman NT, Wang EW, Shin SS, Chang YF, FernandezMiranda JC, Snyderman CH, et al: Does lumbar drainage reduce postoperative cerebrospinal fluid leak after endoscopic endonasal skull base surgery? A prospective, randomized controlled trial. J Neurosurg 131:1172-1178, 2018

\section{Disclosures}

The authors report no conflict of interest concerning the materials or methods used in this study or the findings specified in this paper.

\section{Author Contributions}

Conception and design: Rutland, Delman. Acquisition of data: Rutland, Sohet, Delman. Analysis and interpretation of data: Rutland, Govindaraj, Iloreta, Bederson, Shrivastava, Delman Drafting the article: Rutland. Critically revising the article: Rutland, Gill, Shrivastava, Delman. Reviewed submitted version of manuscript: all authors. Approved the final version of the manuscript on behalf of all authors: Rutland. Statistical analysis: Gill. Study supervision: Shrivastava, Delman.

\section{Correspondence}

John W. Rutland: Icahn School of Medicine at Mount Sinai, New York, NY. jack.rutland@icahn.mssm.edu. 